\title{
Single-shot femtosecond x-ray diffraction from randomly oriented ellipsoidal nanoparticles
}

\author{
M. J. Bogan, ${ }^{1, *}$ S. Boutet, ${ }^{2}$ A. Barty, ${ }^{3,4}$ W. H. Benner, ${ }^{3}$ M. Frank, ${ }^{3}$ L. Lomb,${ }^{5}$ R. Shoeman, ${ }^{5}$ D. Starodub,${ }^{1}$ M. M. Seibert, ${ }^{6}$ \\ S. P. Hau-Riege, ${ }^{3}$ B. Woods, ${ }^{3}$ P. Decorwin-Martin, ${ }^{1}$ S. Bajt, ${ }^{7}$ J. Schulz,${ }^{4}$ U. Rohner, ${ }^{3,8}$ B. Iwan, ${ }^{6}$ N. Timneanu, ${ }^{6}$ \\ S. Marchesini, ${ }^{9}$ I. Schlichting, ${ }^{5}$ J. Hajdu, ${ }^{6}$ and H. N. Chapman ${ }^{4,10}$ \\ ${ }^{1}$ Stanford PULSE Institute, SLAC National Accelerator Laboratory, Menlo Park, California 94025, USA \\ ${ }^{2}$ LCLS, SLAC National Accelerator Laboratory, Menlo Park, California 94025, USA \\ ${ }^{3}$ Lawrence Livermore National Laboratory, Livermore, California 94550, USA \\ ${ }^{4}$ Center for Free-Electron Laser Science, DESY, Notkestrasse 85, 22607 Hamburg, Germany \\ ${ }^{5}$ Max Planck Institute for Medical Research, Heidelberg, Germany Germany, \\ and Max Planck Advanced Study Group, Center for Free-Electron Laser Science, DESY, Hamburg, Germany \\ ${ }^{6}$ Laboratory of Molecular Biophysics, Department of Cell and Molecular Biology, Uppsala University, Uppsala, Sweden \\ ${ }^{7}$ Photon Science, DESY, Notkestrasse 85, 22607 Hamburg, Germany \\ ${ }^{8}$ TOFWERK AG, Thun, Switzerland \\ ${ }^{9}$ Lawrence Berkeley National Laboratory, Berkeley, California 94720, USA \\ ${ }^{10}$ University of Hamburg, Luruper Chaussee 149, 22761 Hamburg, Germany
}

(Received 30 November 2009; published 14 September 2010)

\begin{abstract}
Coherent diffractive imaging of single particles using the single-shot "diffract and destroy" approach with an x-ray free electron laser (FEL) was recently demonstrated. A high-resolution low-noise coherent diffraction pattern, representative of the object before it turns into a plasma and explodes, results from the interaction of the FEL with the particle. Iterative phase retrieval algorithms are used to reconstruct twodimensional projection images of the object from the recorded intensities alone. Here we describe the first single-shot diffraction data set that mimics the data proposed for obtaining 3D structure from identical particles. Ellipsoidal iron oxide nanoparticles $(250 \mathrm{~nm} \times 50 \mathrm{~nm})$ were aerosolized and injected through an aerodynamic lens stack into a soft $\mathrm{x}$-ray FEL. Particle orientation was not controlled with this injection method. We observed that, at the instant the x-ray pulse interacts with the particle, a snapshot of the particle's orientation is encoded in the diffraction pattern. The results give credence to one of the technical concepts of imaging individual nanometer and subnanometer-sized objects such as single molecules or larger clusters of molecules using hard x-ray FELs and will be used to help develop robust algorithms for determining particle orientations and 3D structure.
\end{abstract}

DOI: 10.1103/PhysRevSTAB.13.094701

PACS numbers: 61.05.cp, 42.55.Vc, 42.30.Wb, 42.30.Rx

\section{INTRODUCTION}

The first free electron laser (FEL) reaching into the soft $\mathrm{x}$-ray regime is the FLASH facility at DESY in Hamburg [1]. Operational since 2006, this facility has provided the opportunity for the development of ultrafast imaging experiments using the "diffract and destroy" approach with $\mathrm{x}$-ray wavelengths from 7 to $32 \mathrm{~nm}[2,3]$. The femtosecond diffractive imaging experiment by Chapman et al. [4] proved that diffraction-limited resolution images could be obtained from the exposure of nanostructured nonperiodic objects to single FLASH pulses. The key to recording the diffraction patterns was a specially designed camera containing a laterally graded multilayer mirror that reflects the diffraction pattern onto a CCD camera and allows the intense FEL pulse to pass through a hole [5]. More recently, FLASH experiments have been used to measure the single-pulse coherent diffraction from nanostructured nonperiodic objects $[4,6-8]$ and free-flying aerosols $[9,10]$.

*mbogan@slac.stanford.edu
Iterative phase retrieval algorithms are used to reconstruct images of the objects from the diffraction patterns [11].

The spatial resolution of single particle coherent $\mathrm{x}$-ray diffractive imaging (CXDI) at FLASH is determined in part by the available wavelength range and has dictated the use of sample handling capabilities tuned for objects in the size range of about $50 \mathrm{~nm}$ to $3 \mu \mathrm{m}$. This size range is well covered by many different types of commercially available spherical size-monodisperse standards, such as polymerbased spheres commonly used in biomedical diagnostics, chromatography, and aerosol science. Imaging spheres on a stationary support has played a key role in the development of CXDI experiments at FLASH. A continuum model for CXDI experiments performed at FLASH [12] and femtosecond time-resolved measurements of nanoscale dynamics [8] show that irradiated $140 \mathrm{~nm}$ diameter spheres maintain their integrity during 25 fs pulses. These measurements were facilitated by the size monodispersity of the spheres, which was enhanced by a differential mobility method [13].

The first experimental evidence of single particle FLASH CXDI shortly followed. Image reconstruction of 
individual $145 \mathrm{~nm}$ diameter spheres supported by a $20 \mathrm{~nm}$ thick silicon nitride membrane was performed with the aid of a strong-scattering reference [6]. Use of a strong scattering wave from a gold nanoparticle to amplify a weak scattering wave from a biomolecule or bionanostructure (via heterodyne amplification) injected as a freestanding particle has been proposed as a method for single biomolecule imaging [14]. Motivating factors for the use of sample handling systems free of supporting membranes include the necessity for an isolated sample to achieve adequate oversampling and the elimination of spurious signal contributions due to substrate scattering.

Delivery of freestanding particles for CXDI has been demonstrated using aerodynamic focusing methods developed to deliver aerosols, nanoparticles, viruses, cells, and biomolecules from ambient conditions into vacuum [9,10,15] (Fig. 1). Based on work by Liu et al. [16,17], the apparatus implements in-line thin plate orifices to manipulate the particle lateral spatial distribution prior to them passing through a nozzle and subsequently undergoing supersonic expansion into vacuum. An axisymmetric stack of these thin plate orifices, or aerodynamic lenses, provides successive contractions of a flowing particle beam cross section and enables focusing of a wide range of particle sizes $(1 \mathrm{~nm}$ to $10 \mu \mathrm{m})$ [18]. Equipped with a pressure flow reducer, the inlet of the aerodynamic lens stack samples aerosolized particles from atmospheric pres- sure at a rate of about one liter per minute and injects them into a vacuum chamber to meet an FEL pulse. Aerosolized polystyrene spheres were used to determine optimum pressures to focus particles of diameter $70 \mathrm{~nm}$ to $2 \mu \mathrm{m}$, to measure particle injection rate into vacuum with a single particle charge detector and to visualize the particle beam alignment with a laser [15]. Any atmospheric pressure aerosol generation method can be coupled to the inlet of the aerodynamic lens stack.

A new era of research with hard x-ray free electron lasers (XFELs) has been recently initiated when the Linac Coherent Light Source (LCLS) at the SLAC National Accelerator Laboratory began delivery of photons to experimental stations in the fall of 2009. Extending the "diffract and destroy" approach with hard-x-ray FELs such as LCLS is anticipated to facilitate atomic resolution imaging of nanometer-to-micrometer-sized objects without the need for crystallization $[2,19,20]$.

Here we discuss the general methodology for capturing single particle diffraction from randomly injected particles at XFELs, focusing on an example from FLASH where we captured single-shot diffraction patterns of ellipsoidal iron oxide nanoparticles in random orientations.

\section{EXPERIMENTAL}

Random injection of aerosols through an aerodynamic lens stack perpendicular to a pulsed laser is an experiment

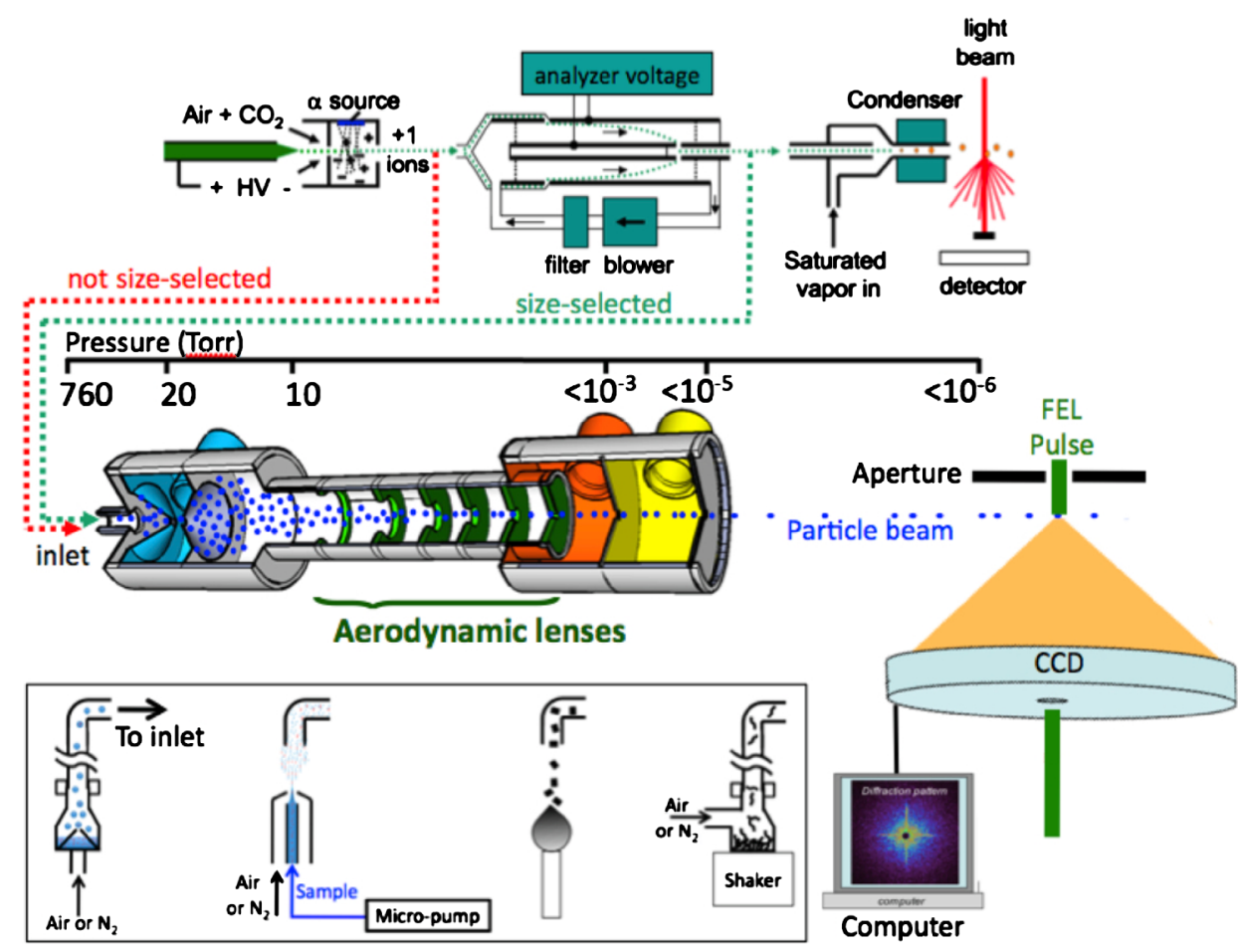

FIG. 1. Conceptual schematic of single-pulse x-ray scattering from single particles in a substrate-free manner. In one variation, electrosprayed spherical nanoparticles are size selected by differential mobility analysis prior to introduction to the differentially pumped aerodynamic lens stack. The particle beam is steered into the FEL and single-pulse diffraction patterns are recorded on a CCD. Alternative aerosol sources tested include nebulizers, atomizers, flames, and dispersion of dry powders. 
most commonly performed as single particle aerosol mass spectrometry (SPAMS) [21-23]. The effective time a particle is present in the laser interaction region, or effective interaction time $\left(t_{\text {eff }}\right)$, is set by the particle's velocity, the diameter of the laser focus, and the laser pulse length. The laser diameter in SPAMS experiments sets $t_{\text {eff }}$ for a particle moving at $150 \mathrm{~m} / \mathrm{s}$ to a few microseconds (Fig. 2), about $10^{3}$ times longer than the typically $3 \mathrm{~ns}$ pulse length of the laser used for desorption/ionization. This has facilitated the use of in situ microparticle tracking systems to trigger a desorption/ionization laser when the particle arrives in the interaction region because timing of particle arrival only needs to be accurate to a couple microseconds. A $100 \%$ hit rate for tracked particles can be achieved using this scheme. Ions generated by the laser/particle interactions are recorded as a mass spectrum after each laser pulse is fired.

The experimental setup for collecting single particle diffraction using ultrafast $\mathrm{x}$-ray lasers is very similar to SPAMS. Ability to capture diffraction from a single particle relies on the coincidence of three events: (1) particle presence in the interaction region, (2) FEL pulse in the interaction region, and (3) CCD data acquisition during a coincidence of (1) and (2). Some key differences are the use of a detector to capture photons scattered off the target particle, the lasers for single particle diffraction have ultrashort pulse lengths, typically $<50 \mathrm{fs}$, and a much smaller focus, resulting in a shorter $t_{\text {eff }}$ (Fig. 2). For instance, the FLASH diameter of $30 \mu \mathrm{m}$ at the interaction region translates to a $t_{\text {eff }}$ in the range of hundreds of nanoseconds for a particle moving at $150 \mathrm{~m} / \mathrm{s}$. In this case $t_{\text {eff }}$ is over $10^{6}$ times longer than the ultrafast FEL pulses, typically $<20$ fs from FLASH, and only an order of

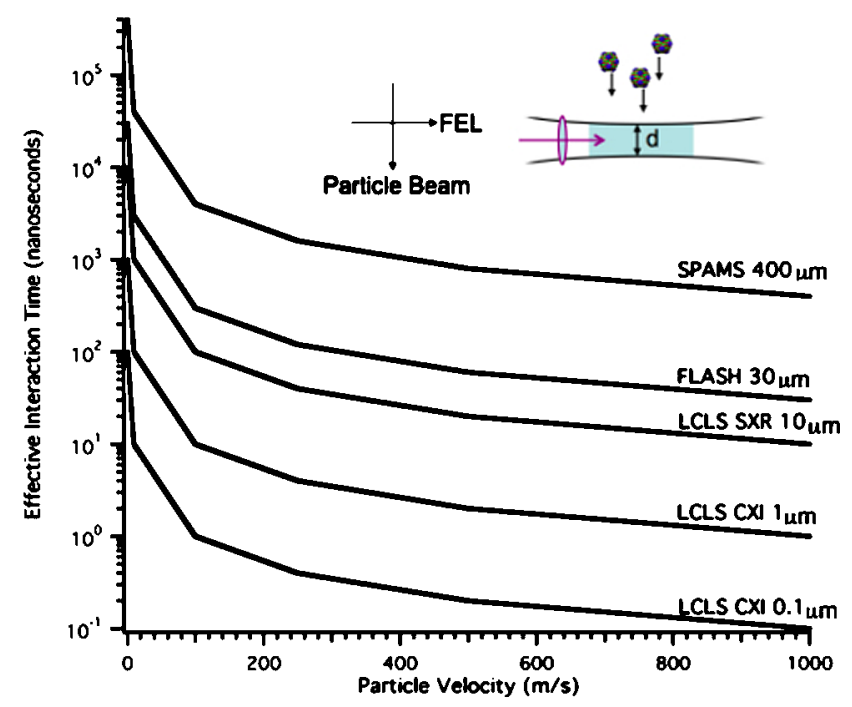

FIG. 2. Estimated effective interaction time, $t_{\text {eff }}$, in a perpendicular geometry, as a function of velocity for particles passing through laser interaction regions with diameter $d$. magnitude shorter than in a SPAMS experiment. The hard $\mathrm{x}$-rays from the LCLS are designed to have a narrower focus than FLASH, from $10 \mu \mathrm{m}$ at first operation down to $100 \mathrm{~nm}$ anticipated for single-molecule imaging on the coherent x-ray imaging (CXI) endstation [24]. At CXI, $t_{\text {eff }}$ is less than $1 \mathrm{~ns}$ for a molecule moving at $150 \mathrm{~m} / \mathrm{s}$. These estimates suggest that a highly tuned SPAMS-based tracking system could possibly send a signal to trigger the XFEL to fire when a particle arrives at the interaction region, but only if a large focus can be used for the data acquisition. No XFEL is currently capable of a triggered mode of operation so single particle diffraction using this type of aerodynamic lens stack relies on random injection.

For random particle injection the instantaneous particle density in the interaction region is most critical. Under identical particle density and x-ray focus conditions, the hit rate for randomly injected particles at FELs is directly proportional to the pulse rate. Current and planned XFEL facilities do not operate with identical pulse rates so the data acquisition rate during single particle diffraction experiments with randomly injected particles at each facility will vary (Table I).

The right bottom panel in Fig. 3 shows a scanning electron micrograph of the test sample, ellipsoidal iron oxide nanoparticles (Corpuscular, NY). The particles have dimensions of $200 \pm 8 \mathrm{~nm}$ by $50 \pm 2 \mathrm{~nm}$, as determined by electron microscopy, and are thus essentially identical within the diffraction-limited resolution of our experimental setup. Aerosols of these particles were generated by two methods. In an atomizer, a gas is used to aspirate the liquid into a (usually) sonic velocity gas jet, wherein it is sheared into droplets. In a nebulizer, this liquid/gas is impacted against a barrier to remove the larger fraction of the droplets. Disposable nebulizers (Salter Labs, Arvin, CA) were used to aerosolize 2-5 $\mathrm{ml}$ of solutions containing nanoparticles with a stream of nitrogen gas (flow rate of 1.0-2.5 L/ min ). A concentric atomizer was constructed from a positive displacement micropump, teflon high-pressure liquid chromatography (HPLC) tubing, and the capillary/sheath nitrogen assembly from a mass spectrometer electrospray ionization (ESI)

TABLE I. Relative hit rate for randomly injected samples with existing and projected XFEL facility pulse parameters, assuming an identical $\mathrm{X}$-ray focus.

\begin{tabular}{lcccc}
\hline \hline & $\begin{array}{c}\text { Maximum } \\
\text { Pulse } \\
\text { separation } \\
(\mu \mathrm{s})\end{array}$ & $\begin{array}{c}\text { pulses per } \\
\text { bunch } \\
\text { (estimated) }\end{array}$ & $\begin{array}{c}\text { Maximum } \\
\text { pulses/sec }\end{array}$ & $\begin{array}{c}\text { Relative } \\
\text { hit rate }\end{array}$ \\
\hline FLASH & 1,10 & 800 & 4000 & 1 \\
LCLS & 8300 & 1 & 120 & 0.03 \\
European XFEL & 0.2 & 300 & 30000 & 7.5 \\
\hline \hline
\end{tabular}




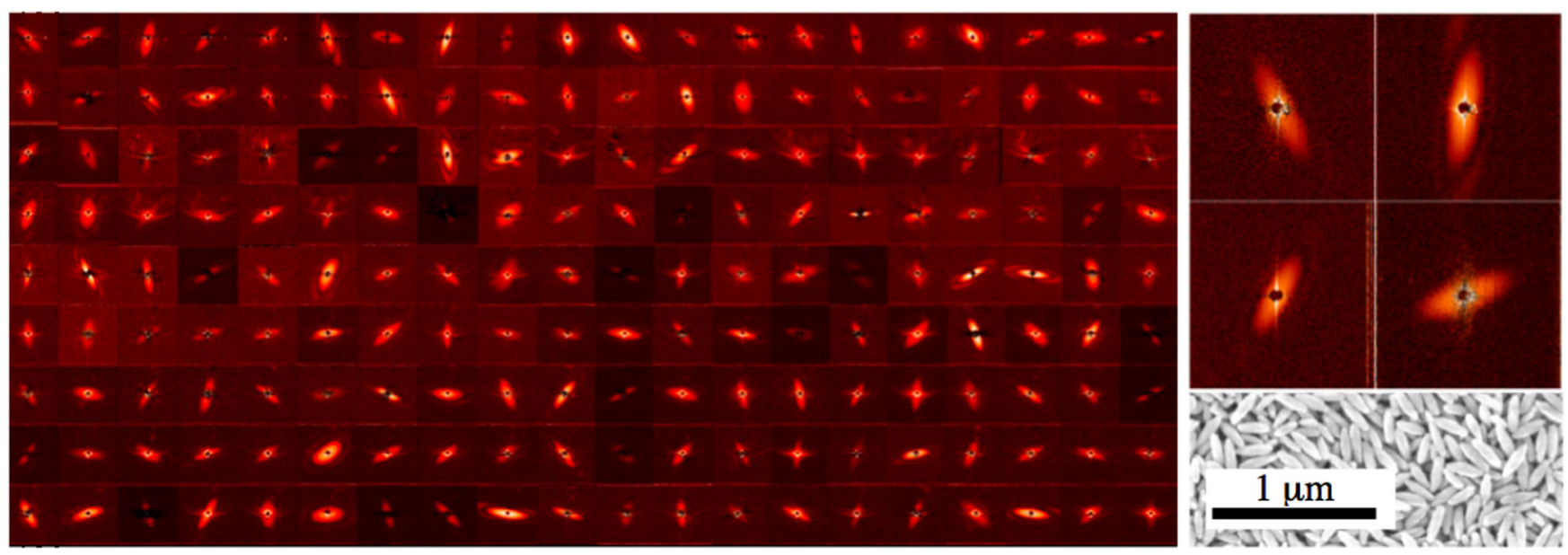

FIG. 3. Diffraction patterns of 180 different single particles (ellipsoidal $50 \mathrm{~nm} \times 200 \mathrm{~nm}$ iron oxide nanoparticles) in unknown random orientations collected at FLASH, $7 \mathrm{~nm}$ wavelength, $10 \mathrm{fs}, 10^{12} \mathrm{ph} /$ pulse. Magnified versions of four diffraction patterns are shown on the top right. Scanning electron micrograph of particles (bottom right).

source. The micropump (mzr-2542 pump with a 19:1 reduction gear) was a biocompatible version (ceramic and PEEK contact elements) and was operated using a SND controller (HNP Mikrosysteme GmbH). The capillary (100 $\mu \mathrm{m}$ inner diameter)/sheath nitrogen assembly for a model 2010A ESI source (Shimadzu Biotech) was connected to the pump (on the input) and neutralization chamber of a model 3780 electrospray aerosol generator (TSI Inc.) (on the output) with standard HPLC connectors and inert tubing of various internal diameters and lengths.

Since a small but visible percentage of the ellipsoidal iron oxide nanoparticles stuck to the tubing interior walls, a new piece of tubing was used for each type of particle. Additionally, the pump was used only with distilled water as the displacement fluid: the pump and tubing were filled with water and the sample was loaded by placing the tubing outlet in the sample and pumping backwards until the desired amount of sample was in the tubing. The pump was stopped and the tubing outlet was connected to the connector on the capillary/nitrogen sheath assembly. Aerosols were produced by starting both the nitrogen sheath flow (approximately 25 psi pressure) and pumping in the forward direction at 20-100 $\mu 1$ per min, depending on the sample. The liquid flow rate was adjusted empirically to maximize the hit rate.

The x-ray camera [5] had a laterally graded multilayer mirror, which reflected the diffraction pattern onto a CCD detector at a distance of $54.9 \mathrm{~mm}$ from the specimen. The mirror worked as a bandpass filter for both wavelength and scattering angle, and isolated the desired scattering pattern from incoherent and plasma emission arising from the sample, and from nonsample-related scattering. A hole in the center of the mirror allowed the direct beam to pass harmlessly through the instrument into a beam dump at a safe distance behind the instrument.

\section{RESULTS AND DISCUSSION}

\section{A. Single particle diffraction of ellipsoidal nanoparticles}

A representative set of single-shot diffraction patterns from 180 individual particles is shown in Fig. 3. At the instant the x-ray pulse interacts with the particle, a snapshot of the particle's orientation is encoded in the diffraction pattern. These particles were chosen because they strongly scatter and their diffraction patterns would provide clear evidence of their relative orientation. This is the first single-shot femtosecond $\mathrm{x}$-ray diffraction data set ever recorded of identical single particles in different unknown random orientations. This type of data set has been proposed for determining three-dimensional structures of identical particles using iterative phase retrieval [2].

Figure 4(A) shows an example of the diffraction pattern acquisition rate for randomly injected ellipsoidal nanoparticles. FLASH was operating with 100 pulses per bunch or 500 pulses per second total. This diffraction pattern acquisition rate was typical for this particular combination of aerodynamic lens stack and nebulizer with nanoparticle solutions of about $10^{12}$ particles $/ \mathrm{ml}$. Since data acquisition from random injection follows Poisson statistics, a target of $\sim 60 \%$ single particle diffraction events was chosen to maximize single particle diffraction while minimizing the number of multiple particle diffraction events. Figure 4(B) shows an example of a diffraction pattern resulting from two different particles being hit with two different pulses while the camera is integrating. Random particle injection will always be plagued by multiparticle scattering events as the limit of $100 \%$ detected particle diffraction events is approached.

For the data in Fig. 4(A), the detector integration and readout time were equal, $1 \mathrm{~s}$ for each. Recall that a diffraction pattern of a single particle will only be recorded 

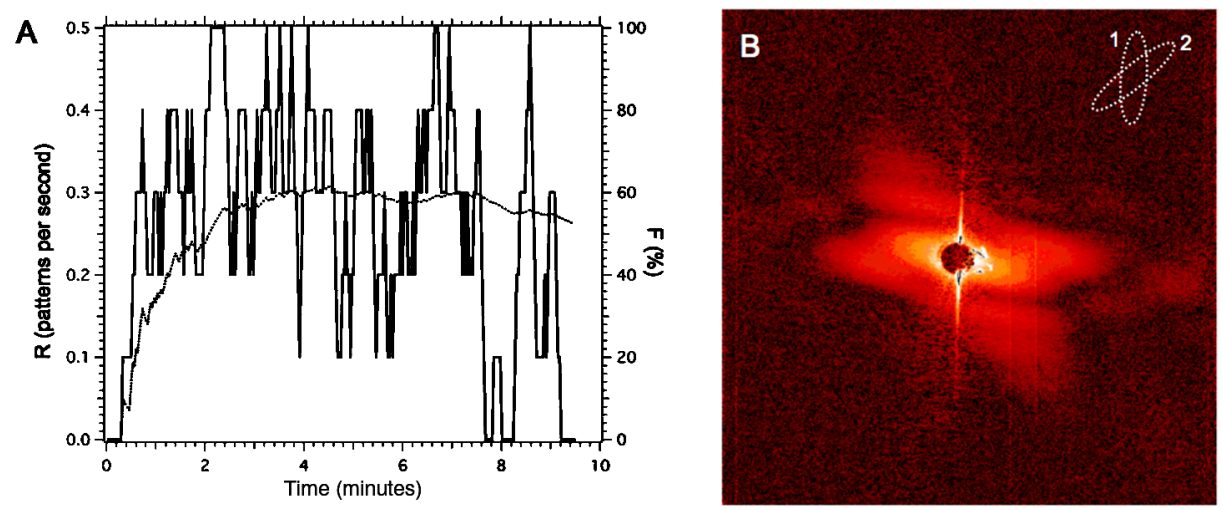

FIG. 4. (A) The instantaneous single particle diffraction acquisition rate $R$ (solid line, 10 s running average) and the cumulative percentage of total camera readouts with evidence of single particle diffraction $F$ for a 10 min experiment with nebulized ellipsoidal nanoparticles (149 diffraction events in 285 camera readouts). FLASH frequency $=500$ pulses s $^{-1}$. The camera integrated signal from 500 pulses and then readout for $1 \mathrm{~s}$, setting a maximum on the single particle diffraction acquisition rate to $0.5 \mathrm{~Hz}$. Experimental conditions are typically set so $F$ (dotted line) reaches $60 \%$ to stay in the single particle diffraction regime. (B) Example diffraction pattern of two different particles hit on two different pulses and detected during a single camera integration/readout cycle. The inset speculates the relative orientation of the two particles (not accounting for out of plane rotation).

when the particle arrival into the FEL interaction region coincides with FEL pulse arrival and detector integration. Thus, $50 \%$ of the random particle arrival and FEL overlaps were not recorded and the maximum data acquisition rate was limited to 0.5 patterns/second while the actual particle/ FEL coincidence rate is 1 per second or more. Specialized detectors with higher framing rates are being developed to remove this limitation [25]. The acquisition of 0.5 patterns per second rate is an order of magnitude larger than achieved previously [9]. Further optimized aerodynamic lens stacks and more efficient aerosolization methods coupled with faster detectors is anticipated to increase the single particle diffraction data acquisition rate at FLASH by at least another 2 orders of magnitude.

\section{B. Projection images "Free" ellipsoidal nanoparticles}

Iterative phase retrieval from single-shot FLASH diffraction patterns of fixed targets and single particles injected as a particle beam to recover the object's electron density is well established for $32,13.5$, and $7 \mathrm{~nm}$ wavelengths from FLASH. Using the Shrinkwrap algorithm [11], the projection images of injected ellipsoidal nanoparticles were recovered within a few thousand iterations. Figure 5(A) shows the background subtracted single-shot diffraction pattern and reconstructed image of a single ellipsoidal particle. As demonstrated for nanoscale polystyrene spheres [10], aerosol generation conditions can be modified to create clusters of the ellipsoidal nanoparticles. For the particular cluster imaged in Fig. 5(B), the particles stacked side by side. Such clusters are commonly observed when the aerosolized particles are captured on a substrate and viewed by scanning electron microscopy. The projection images in Fig. 5 are the averaged result of five recon- structions using random starting conditions. The repeatability of the recovered images as a function of resolution measures the effective phase retrieval transfer function (PRTF) and the PRTF half-period resolution, in this case $35 \mathrm{~nm}$, is commonly quoted as the resolution of the reconstructed image.
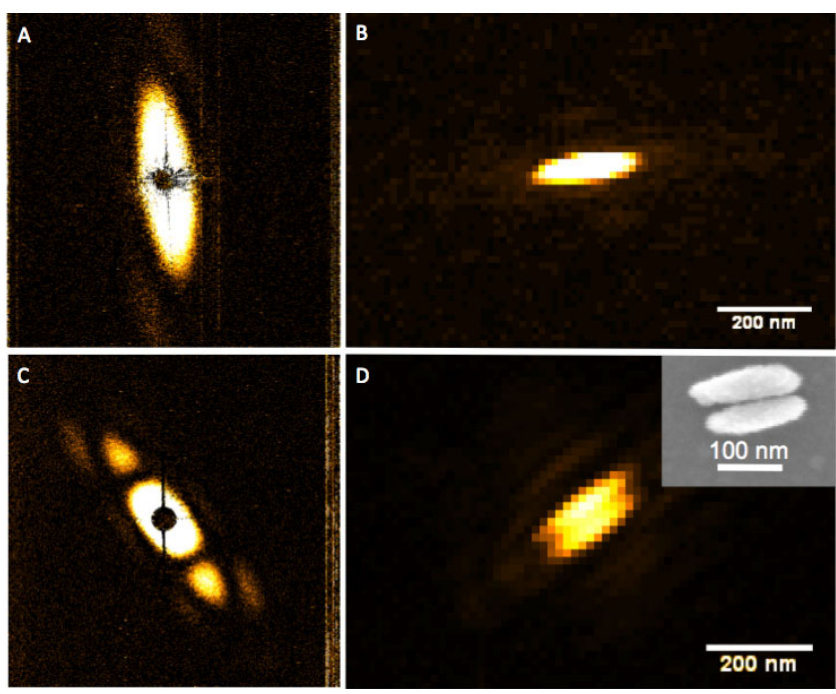

FIG. 5. Single-shot diffraction patterns (FLASH, $7 \mathrm{~nm}, 10 \mathrm{fs}$, $10^{12} \mathrm{ph} / \mathrm{pulse}$ ) and corresponding reconstructed electron density (both linear scales) of (A)-(B) one or (C)-(D) two ellipsoidal iron oxide nanoparticles. In both diffraction patterns the central lobe and a faint first side lobe are detectable against the background. The lack of further side lobes results in weak artifacts in the reconstruction. Pixel dimensions are about $14 \mathrm{~nm}$ by $15 \mathrm{~nm}$ for (B) and (D), respectively. Inset: Scanning electron microscopy of nebulized particles captured on a silicon wafer, $100 \mathrm{k}$ magnification. 


\section{Towards 3D image reconstructions}

To use iterative phase retrieval to recover the 3D structure, particle orientation must be determined for each diffraction pattern. One approach is the use of spatial correlation analysis of scattered intensity fluctuations applied to the complete data set of all diffraction patterns in order to recover a 3D diffraction volume. This method was first developed for solution scattering from many particles [26], and recently adopted to a single-molecule XFEL diffraction experiment [27]. While a simple sum of individual diffraction intensities results in a spherically averaged diffraction pattern, producing the same type of data as in small angle $\mathrm{x}$-ray scattering (SAXS), averaging of the products of scattered intensities in different pixels enhances the scattering from the single particle, thus revealing information additional to SAXS data. The major challenge in this method is calculation of the coefficients $S_{l m}(k)$ in the spherical harmonics expansion of scattered intensity $S(\mathbf{k})$ from the equation $C_{l}\left(k_{1} k_{2}\right)=\sum_{m} S_{l m}\left(k_{1}\right) S_{l m}^{*}\left(k_{2}\right)$, where $C_{l}\left(k_{1} k_{2}\right)$ is an $N \times N$ matrix, directly obtained from the experimental data binned to a grid of $N$ samples of the absolute value of the momentum transfer vector. The special solutions for $S_{l m}(k)$ can be easily obtained, but since equations for different $l$ are independent, a special effort is required to relate the coefficients corresponding to different values of $l$. In principle, this can be done by involving higher order correlations [28]. The situation is greatly simplified in the case of ellipsoidal iron oxide nanoparticles, which possess an axis of rotation. If this axis is aligned with the $z$ axis, then only expansion coefficients $S_{l 0}(k)$ with $m=0$ will have nonzero values. Using coefficients $S_{l m}(k)$ calculated up to a required resolution, the 3D distribution of scattered intensity on a cubic grid can be constructed. Subsequently, iterative phase retrieval algorithms can be applied to reconstruct the object electron density. Application of the spatial correlation approach to single particle diffraction using ultrafast $\mathrm{x}$-ray pulses reduces experimental difficulties associated with implementation of this method to solution scattering using traditional $\mathrm{x}$-ray sources. First, it eliminates the background of solvent and spherically averaged particle scattering, inevitable in solution scattering. Second, as the right panel of Fig. 3 clearly demonstrates, a single pulse provides more than adequate scattered intensity from a particle "frozen" in space. In contrast, in solution scattering experiments using conventional synchrotron x-ray sources, it can be problematic to choose the exposure time so it is long enough to achieve several scattering events from the same particle and short enough for particles to rotate only a small angle determined by required resolution.

\section{CONCLUSION}

Femtosecond $\mathrm{x}$-ray diffraction from randomly injected freestanding ellipsoidal nanoparticles was collected at FLASH $(7 \mathrm{~nm}$ wavelength, 10 fs pulse length,
$10^{12}$ photons/pulse). This is the first single-shot diffraction data set that mimics the kind of "diffract and destroy" data proposed to be necessary to obtain 3D structure of injected particles from XFELs. The results give credence to one of the technical concepts of imaging individual nanometer and subnanometer-sized objects such as single molecules or larger clusters of molecules using hard x-ray FELs. Note that, during the review of this manuscript, we have used portions of this data set to test the EMC algorithm for determining particle orientations and 3D structure [29].

\section{ACKNOWLEDGMENTS}

This work was supported by the following agencies: M. J.B., D. S., and P.D. M. were supported through the Stanford PULSE Institute at the SLAC National Accelerator Laboratory by the U.S. Department of Energy, Office of Basic Energy Sciences; The U.S. Department of Energy in part under Contract No. DEAC02-76SF00515 and Lawrence Livermore National Laboratory in part under Contract No. W-7405-Eng-48 and in part under Contract No. DE-AC52-07NA27344, Lawrence Livermore National Laboratory (the project 05-SI-003 from the Laboratory Directed Research and Development Program of LLNL); the Deutsches Elektronen-Synchrotron, a research center of the Helmholtz Association. We acknowledge the financial support of the Sven and Lilly Lawski Foundation to M. M. S. Additional support comes from the DFG Cluster of Excellence at the Munich Centre for Advanced Photonics [30], from the Virtual Institute Program of the Helmholtz Society, the Max Planck Society, and by the Swedish Research Council. The Advanced Light Source is supported by the Director, Office of Science, Office of Basic Energy Sciences, of the U.S. Department of Energy under Contract No. DE-AC02-05CH11231.

[1] W. Ackermann et al., Nat. Photon. 1, 336 (2007).

[2] G. Huldt, A. Szoke, and J. Hajdu, J. Struct. Biol. 144, 219 (2003).

[3] K. E. Schmidt et al., Phys. Rev. Lett. 101, 115507 (2008).

[4] H. N. Chapman et al., Nature Phys. 2, 839 (2006).

[5] S. Bajt et al., Appl. Opt. 47, 1673 (2008).

[6] S. Boutet et al., J. Electron Spectrosc. Relat. Phenom. 166-167, 65 (2008).

[7] A. Barty et al., Nat. Photon. 2, 415 (2008).

[8] H. N. Chapman et al., Nature (London) 448, 676 (2007).

[9] M. J. Bogan et al., Nano Lett. 8, 310 (2008).

[10] M. J. Bogan et al., Aerosol Sci. Technol. 44, 1 (2010).

[11] S. Marchesini, Rev. Sci. Instrum. 78, 011301 (2007).

[12] S. P. Hau-Riege, R. A. London, H. N. Chapman, and M. Bergh, Phys. Rev. E 76, 046403 (2007).

[13] M. J. Bogan et al., J. Aerosol Sci. 38, 1119 (2007).

[14] T. Shintake, Phys. Rev. E 78, 041906 (2008).

[15] W. H. Benner et al., J. Aerosol Sci. 39, 917 (2008). 
[16] P. Liu et al., Aerosol Sci. Technol. 22, 314 (1995).

[17] P. Liu et al., Aerosol Sci. Technol. 22, 293 (1995).

[18] A.S. Wexler and M. V. Johnston, Real-Time SingleParticle Analysis (John Wiley \& Sons, Hoboken, New Jersey, 2005), p. 365.

[19] R. Neutze et al., Nature (London) 406, 752 (2000).

[20] J.C.H. Spence and R.B. Doak, Phys. Rev. Lett. 92, 198102 (2004).

[21] D. G. Nash, T. Baer, and M. V. Johnston, Int. J. Mass Spectrom. 258, 2 (2006).

[22] P. T. Steele et al., Anal. Chem. 80, 4583 (2008).

[23] M. J. Bogan et al., Rapid Commun. Mass Spectrom. 21, 1214 (2007).
[24] S. Boutet and G. Williams, New J. Phys. 12, 035024 (2010).

[25] L. Struder et al., Nucl. Instrum. Methods Phys. Res., Sect. A 614, 483 (2010).

[26] Z. Kam, Macromolecules 10, 927 (1977).

[27] D. K. Saldin et al., J. Phys. Condens. Matter 21, 134014 (2009).

[28] Z. Kam and I. Gafni, Ultramicroscopy 17, 251 (1985).

[29] N.T.D. Loh et al., Phys. Rev. Lett. 104, 225501 (2010).

[30] http://www.munich-photonics.de 